Supporting Information for

\title{
Biodegradable gold nanoclusters with improved excretion due to pH-trig- gered hydrophobic-to-hydrophilic transition
}

\author{
Elizabeth M. Higbee-Dempsey, ${ }^{1}$ Ahmad Amirshaghaghi, ${ }^{2}$ Matthew J. Case, ${ }^{3}$ Mathilde Bouché, ${ }^{4}$ Johoon Kim, ${ }^{4}$ \\ David P. Cormode, ${ }^{4}$ and Andrew Tsourkas. ${ }^{*}, 2$ \\ ${ }^{1}$ Biochemistry and Molecular Biophysics Graduate Group, Perelman School of Medicine, ${ }^{2}$ Department of Bioengineering, ${ }^{3}$ Depart- \\ ment of Radiation Oncology, and ${ }^{4}$ Department of Radiology, University of Pennsylvania, Philadelphia, PA 19104.
}

\section{EXPERIMENTAL PROCEDURES}

Materials: Dextran (5 kDa, T5) was purchased from Phamacosmos. 3,3'-dithiobis(propanoic dihydrazide) was purchased from Frontier Scientific. Tris(2-carboxyethyl(phosphine) (TCEP), gold(III) chloride trihydrate, tetraoctylammonium bromide, 1-dodecanethiol, sodium borohydride, pyridinium-p-toluenesulfonate, and 2-methoxypropene were purchased from Sigma Aldrich. $p$-mercaptobenzoic acid ( $p$ MBA) was purchased from TCI America. Sodium cyanoborohydride, triethylamine, fetal bovine serum (FBS), Dulbecco's Modified Eagle Medium (DMEM), Trypsin-EDTA, and penicillin-streptomycin solution were purchased from Thermo Fisher Scientific. Poly(ethylene glycol $)_{4000}-b$-poly $(\varepsilon$-caprolactone $)_{3000}$ (PEG-PCL) was purchased from Polymer Source. PBS (1X, pH 7.4) was purchased from Quality Biological. MTT assay kit was purchased from Roche Diagnostics $\mathrm{GmbH}$. Isoflurane was purchased from Akorn Animal Health.

Synthesis of pMBA-AuNPs: Hydrophilic gold nanoparticles coated in $p$-mercaptobenzoic acid ( $p$ MBA) were prepared using a protocol modified from Jadzinsky et al. ${ }^{1}$ Briefly, a stock solution of gold(III) chloride trihydrate $\left(1 \mathrm{~g} \mathrm{~mL}^{-1}\right)$ was first centrifuged for 30 minutes at $16,000 \mathrm{x} g$ to remove gold crystals, and then $400 \mu \mathrm{L}$ was added to an acid-washed flask and diluted in water $(158 \mathrm{~mL}) .184 \mathrm{~mL}$ methanol was then added under vigorous stirring. In parallel, $p \mathrm{MBA}$ was prepared as a $95 \mathrm{mM}$ stock solution $(726 \mathrm{mg}$ in $50 \mathrm{~mL})$ containing $300 \mathrm{mM}$ sodium hydroxide $(595 \mathrm{mg})$, and then combined with the primary gold solution. The resulting solution was allowed to equilibrate for 3-5 hours, until the color had changed from pale orange to nearly colorless. Next, a freshly-prepared $150 \mathrm{mM}$ solution of sodium borohydride (159 mg in $28 \mathrm{~mL}$ water) was added to the reaction dropwise over the course of 15 minutes, resulting in a gradual color change to brown and then black over the course of 2 hours. The solution continued stirring overnight. Particles were then precipitated by adding methanol $(467 \mathrm{~mL})$ and an aqueous solution of sodium chloride $(6.825 \mathrm{~g}$ in $47 \mathrm{~mL})$ to the reaction mixture, then centrifuging at 3000 
$\mathrm{x} g$ for 5 minutes. To wash particles, pellets were first suspended in $70 \%$ methanol (v/v) and centrifuged again; next, pellets were dissolved in water and rinsed repeatedly using centrifugal filters (10,000 MWCO, Amicon). Finally, particles were added to pre-weighed microcentrifuge tubes and then dried under centrifugal evaporation (Labconco).

Synthesis of thiolated dextran: Thiolated dextran was prepared by reductive amination. ${ }^{2}$ Dextran ( $\left.5 \mathrm{kDa} ; 14.163 \mathrm{~g}\right)$ and 3,3'-dithiobis(propanoic dihydrazide) $(270 \mathrm{mg}$ ) were dissolved in $300 \mathrm{~mL}$ acetate buffer (0.3 M, pH 5). Sodium cyanoborohydride (approximately $2.6 \mathrm{~g}$ ) was then added, and the reaction was allowed to stir for 24 hours. Next, a solution of TCEP (460 mg in $7 \mathrm{~mL}$ water) was brought to $\mathrm{pH} 5$ using sodium hydroxide, then added to the primary reaction solution. After 6 hours stirring, the solvent was removed by lyophilization (Labconco) and the solid product was ground to a fine powder. Thiolated dextran was then purified for characterization using Soxhlet extraction in methanol, then dried under vacuum. Yield: $26 \%(\mathrm{w} / \mathrm{w}) .{ }^{1} \mathrm{H}$ NMR $\left(\mathrm{D}_{2} \mathrm{O}, 360 \mathrm{MHz}\right) \delta 1.90(\mathrm{~s}, 4 \mathrm{H}), 2.10(\mathrm{~m}, 2 \mathrm{H}), 2.39(\mathrm{~m}, 2 \mathrm{H}), 3.34(\mathrm{~m}, 20 \mathrm{H}), 3.50$ (m, 56H), 3.73 (br dd, 62H), $3.92(\mathrm{~m}, 58 \mathrm{H}), 4.97(\mathrm{~m}, 28 \mathrm{H}), 5.32(\mathrm{~m}, 1 \mathrm{H})$.

To prepare thiolated dextran for reaction with $p$ MBA-AuNPs, an alternative purification procedure was utilized. After lyophilizing solvent and grinding the solid product to a fine powder, solids were washed four times by suspension in $90 \%$ methanol (v/v) followed by centrifugation. Between washes, pellets were mechanically disturbed either by agitating with a spatula (after first two washes) or by drying the pellet under vacuum and re-pulverized (after the third wash). Finally, the product was dried under vacuum.

Synthesis of Dextran-AuNPs: pMBA-AuNPs were dissolved in aqueous solution at a concentration of approximately $2 \mathrm{mg} \mathrm{mL}^{-1}$ (by dry nanoparticle weight). Thiolated dextran was added at approximately $10 \mathrm{x}$ molar equivalents relative to the $p$ MBA ligand (1 part $p$ MBA AuNPs : 85 parts Dext-SH,w:w; calculated by approximating AuNP mass percentage at $25 \%$ MBA, and approximating molecular weight of thiolated dextran at $5239 \mathrm{Da}$ ). The reaction was stirred for 48 hours, and then purified by repeated washing using centrifugal filters (30,000 MWCO). Finally, the resulting dextran-coated AuNPs were lyophilized.

Synthesis of AcetalDextran-AuNPs: Dextran-AuNPs were acetalated using a protocol adapted from Bachelder et al. ${ }^{3}$ A 20-mL flask was flame-dried under vacuum and then charged with nitrogen. Meanwhile, Dextran-AuNPs (up to $500 \mathrm{mg}$ ) were dissolved in $10 \mathrm{~mL}$ anhydrous DMSO, then combined with $16 \mathrm{mg}$ pyridinium-p-toluenesulfonate; this was transferred 
to the reaction flask under dry nitrogen using standard Schlenk line techniques. $3.4 \mathrm{~mL}$ of 2-methoxypropene was then injected into the same flask, which was allowed to stir for 3 hours at room temperature under oxygen-free conditions. The reaction was quenched by addition of $1 \mathrm{~mL}$ triethylamine. To purify, the solution was diluted into $100 \mathrm{~mL}$ water, and approximately $10 \%$ volume was removed by rotary evaporation to ensure elimination of excess triethylamine. The solution was then centrifuged at $3000 \mathrm{x} g$ to collect precipitates. Any solids that remained in the original reaction vessel or in the flask used for rotary evaporation were washed with water. Finally, all precipitates were dissolved in acetone, combined, transferred into pre-weighed microcentrifuge tubes, then dried under centrifugal evaporation.

Synthesis of AcetalDextran-AuNP-Micelles: A solution was prepared containing $80 \mathrm{mg} \mathrm{mL}^{-1}$ of Acetal-Dextran-AuNPs (by dry particle weight) and $40 \mathrm{mg} \mathrm{mL}^{-1}$ of poly(ethylene glycol)-block-poly( $\varepsilon$-caprolactone) (PEG $\left.\mathrm{P}_{4000}-\mathrm{PCL}_{3000}\right)$. After thorough sonication, $100 \mu \mathrm{L}$ of this solution was injected slowly into a glass scintillation vial containing $4 \mathrm{~mL}$ water under constant sonication (Branson, bath sonicator); sonication continued until a homogenous emulsion was formed. Vials were lightly capped and placed under vacuum overnight to remove toluene, allowing formation of stable micelles. Micelles were purified by centrifugation, first at $400 \times \mathrm{g}$ (10 minutes) to eliminate large precipitating aggregates and then at $16,000 \times \mathrm{g}(30$ minutes) to sediment micelles. This precipitate was then collected and further concentrated using centrifugal filters (100,000 MWCO). Particles were then stored at $4^{\circ} \mathrm{C}$, typically after suspending in PBS (by adding 10x concentrate).

Synthesis of $C_{12}$-AuNPs and $C_{12}$-AuNP-Micelles: Gold nanoparticles and gold-loaded polymeric micelles were synthesized as non-pH-sensitive experimental controls. Dodecanethiol-coated AuNPs ( $\mathrm{C}_{12}$-AuNPs) were synthesized using the Brust method, ${ }^{4}$ as adapted by Higbee-Dempsey et al. ${ }^{5}$ Dodecanethiol-coated AuNPs were then loaded into polymeric micelles using a protocol modified from Al Zaki et al. ${ }^{6,7}$ PEG-PCL $\left(17 \mathrm{mg} \mathrm{mL}^{-1}\right)$ and dodecanethiol AuNPs $\left(17.5 \mathrm{mg} \mathrm{mL}^{-1}\right.$ by dry particle weight) were dissolved in a total of $200 \mu \mathrm{L}$ toluene. This solution was sonicated and then injected into $4 \mathrm{~mL}$ water to form a homogenous emulsion. Purification then proceeded as described for AcetalDextran-AuNP-Micelles.

Material characterization: Thiolated dextran was characterized via NMR; data was collected on Bruker DMX 300 and 360 NMR spectrometers, and analyzed using ACD Spectrus software. Gold nanoparticles were characterized using a variety of physiochemical methods. Particles were imaged by electron microscopy using a Tecnai T12 microscope to determine gold diameter and overall particle morphology; size analysis was performed using ImageJ software. Hydrodynamic diameter 
and zeta potential were measured using a Zetasizer Nano ZS (Malvern Panalytical); data are generally expressed as the average of triplicate measurements of one synthetic batch; while data for AcetalDextran-AuNP-Micelles represent an average of six batches. Gold concentration was determined using inductively-coupled plasma optical emission spectroscopy (ICPOES) on a Spectro Genesis. To prepare samples for ICP-OES, solutions were placed in borosilicate glass tubes containing up to $1 \mathrm{~mL}$ of freshly-prepared aqua regia (a 1:3 mixture of nitric acid and hydrochloric acid; e.g., $250 \mu \mathrm{L} \mathrm{HNO}_{3}$ and $750 \mu \mathrm{L}$ $\mathrm{HCl}$ ); tubes were lightly sealed with PFTE-lined caps, allowed to stand for at least 20 minutes to ensure complete dissolution of gold, and then diluted in water up to a known volume. For each particle formulation, mass percentage of gold was calculated by dissolving a known mass of dried particle into a known volume of liquid, then performing ICP analysis to determine gold concentration.

Evaluation of acid-responsiveness: AcetalDextran-AuNPs were first dissolved in acetone and then diluted into buffered solutions at one of three pHs: $\mathrm{pH} 7.4$ (PBS); $\mathrm{pH} 6.4$ (PBS, titrated to 6.8 using $1 \mathrm{M}$ sodium hydroxide); or $\mathrm{pH} 5.0$ (acetate buffer, $0.3 \mathrm{M}$ ). All solutions consisted of $1.5 \mathrm{mg} \mathrm{mL}^{-1}$ AuNPs (by dry particle weight) and $2 \%$ acetone (v/v). Solutions were placed at $37^{\circ} \mathrm{C}$ and agitated vigorously using an orbital shaker. Every 1.5 hours, triplicate aliquots were removed and analyzed. Absorbance was measured at $750 \mathrm{~nm}$ (Tecan) to determine solution turbidity, as calculated using the following equation:

$$
\% \text { Transmittance }=\text { antilog }(2-\text { absorbance })
$$

Samples were then diluted to a concentration of $1 \mathrm{mg} \mathrm{mL}^{-1}$ and photographed.

In separate experiments, AcetalDextran-AuNPs and AcetalDextran-AuNP-Micelles were examined in serum. First, fetal bovine serum was sterilely titrated to $\mathrm{pH} 5.0$ using hydrochloric acid; this serum and unmodified serum were equilibrated at $4{ }^{\circ} \mathrm{C}$ overnight, and then centrifuged at $3000 \mathrm{x}$ for 30 minutes to remove any aggregated proteins. Gold particles were then sterilely dissolved in both serums at a concentration of $0.04 \mathrm{mg} \mathrm{mL}^{-1}$ by $\mathrm{Au}$, and divided into two $1.5-\mathrm{mL}$ sterile microfuge tubes per experimental group ( $1 \mathrm{~mL}$ each). One set of tubes were immediately centrifuged at $16,000 \mathrm{x} g$ for 10 minutes, and pellets were photographed; the other set of tubes were incubated at $37^{\circ} \mathrm{C}$ for 24 hours under constant agitation, then similarly centrifuged and photographed. 
Finally, AcetalDextran-AuNP-Micelles were dissolved in buffered solutions at either pH 7.4 (PBS) or pH 5.0 (acetate buffer, $0.3 \mathrm{M}$ ). A small aliquot of particles were immediately removed and diluted 10-fold in millipore water, placed on a TEM grid and allowed to dry, then imaged by TEM (Tecnai T12). The remaining particles in salt buffer were examined by DLS. Solutions were then placed at $37^{\circ} \mathrm{C}$ and agitated vigorously using an orbital shaker. After 24 hours, solutions were again prepped for TEM and DLS as described.

Cell culture: RAW 264.7 and HepG2 cells were acquired from ATCC. Cells were grown at $37^{\circ} \mathrm{C}$ with $5 \% \mathrm{CO}_{2}$ in Dulbecco's Modified Eagle Medium (DMEM) supplemented with $10 \%$ fetal bovine serum and $1 \%$ pen strep. Cell passage numbers were kept low $(<10)$ to prevent genetic drift; during passage, RAW 264.7 cells were dissociated using cell scrapers, while HepG2 cells were dissociated with trypsin-EDTA.

Determination of cell viability: HepG2 human liver cancer cells were examined by MTT assay to determine cell viability. Cells were first seeded in a 96-well plate $\left(1 \times 10^{5}\right.$ cells per well). The following day, media was carefully removed by pipette and replaced with $100 \mu \mathrm{L}$ fresh media containing AcetalDextran-AuNP-Micelles at concentrations ranging from 0 to $80 \mu \mathrm{gLL}^{-1}$ (by Au concentration). Cells were incubated for 24 hours, after which the media was removed and wells were washed with $200 \mu \mathrm{L}$ PBS. Finally, cells were given fresh media containing 3-(4,5-dimethylthiazol-2-yl)-2,5-diphenyltetrazolium bromide (MTT). The MTT assay then proceeded according to the manufacturer's instructions. Cell viability was normalized to untreated control cells, and all conditions were performed in triplicate wells.

Cell uptake: To examine cellular uptake of AcetalDextran-AuNP-micelles, particles were incubated with RAW 264.7 mouse macrophages. Cells were first seeded in 6-well plates $\left(2 \times 10^{6}\right.$ cells per well, quadruplicate conditions). The following day, media was carefully removed by pipette and replaced with $1 \mathrm{~mL}$ fresh media containing micelles at a concentration of $10 \mu \mathrm{g}$ Au per mL. In parallel control experiments, cells were treated with $10 \mu \mathrm{g} / \mathrm{mL} \mathrm{C}_{12}$-AuNP-Micelles. After 24-hours incubation, media was carefully collected by pipette and set aside; cells were then gently washed with $1 \mathrm{~mL}$ PBS, which was also collected. An additional $1 \mathrm{~mL}$ PBS was then added, and adherent cells were gently harvested using cell scrapers.

Cells were then examined using several techniques. In one set of experiments, cells ( 3 wells each) were counted using a hemocytometer, transferred into pre-weighed centrifuge tubes, weighed again, and lyophilized until dry. Dry tubes were 
weighed a final time, and then solids were dissolved in $1 \mathrm{~mL}$ aqua regia and processed for ICP-OES analysis as described. Tube weights were used to calculate exact number and mass of cells.

In another set of experiments, washed cells ( 1 well each) were centrifuged at $500 \mathrm{x} g$ for 5 minutes, and cell pellets were gently resuspended in $100 \mu \mathrm{L}$ of fixative solution containing $2 \%$ glutaraldehyde and $2 \%$ paraformaldehyde (w/v). Cells were then prepared for EM imaging by the University of Pennsylvania Electron Microscopy Resource Lab, and imaged on a JEOL 1010 microscope.

Animal welfare statement: Female C57BL/6 mice (6-8 weeks old) were obtained from Charles River. All animals were housed and maintained according to procedures approved by the University of Pennsylvania Institutional Animal Care and Use Committee. Mice were fed nutritionally standard chow containing fenbendazole, with free access to food and water.

Biodistribution and safety of AcetalDextran-AuNP-Micelles: Mice were intravenously injected with AcetalDextranAuNP-Micelles at a concentration of $100 \mathrm{mg}$ Au per kg body weight ( $n=18$ mice total). Mice were weighed prior to injection and then at a minimum of once per week post-injection. Blood samples were then collected from the opposite tail vein at the following timepoints: 2 hours, 6 hours, 24 hours, and 72 hours. At each timepoint, approximately 10-25 $\mu \mathrm{L}$ were removed from each of three mice, which were randomized such that every mouse underwent no more than 3 blood draws in total. Whole blood samples were then analyzed by ICP-OES as described, by digestion in $1 \mathrm{~mL}$ aqua regia for a minimum of one hour. Half-life of gold in blood samples was determined by fitting a double exponential decay curve to the data using MATLAB software (Mathworks), which yielded the following equation:

$$
y=56.9835^{-0.3133 x}+26.0405^{-0.0439 x}
$$

where $x=$ time post-injection and $y=$ percent injected dose per gram tissue; half-life was calculated with respect to estimated values at 1 minute post-injection. In a separate study, control mice $(n=3$ mice $)$ were intravenously injected with an equivalent volume of $0.9 \%$ saline, and then blood samples were drawn as described at 2 hours, 6 hours, and 24 hours postinjection. Mice were weighed prior to injection and then again on days 1, 4, and 7 post-injection. 
Mice were then sacrificed at various timepoints post-injection: 1 day, 3 days, 1 week, 2 weeks, 4 weeks, and 12 weeks $(n$ $=3$ mice per group). Prior to sacrifice, urine and feces samples (12-130 $\mu \mathrm{L}$ and $18-75 \mathrm{mg}$, respectively) were directly collected from lightly-scruffed mice. Mice were then anesthetized with isoflurane $\left(3 \%, 1.5 \mathrm{~L} \mathrm{~min}^{-1} \mathrm{O}_{2}\right)$, and a minimum of 500 $\mu \mathrm{L}$ blood was collected via cardiac puncture. Mice were euthanized by cervical dislocation, and the following organs were harvested (whole/intact or a fragment thereof): brain, heart, kidney, liver, lung, muscle, skin, and spleen. Organs were lightly rinsed in PBS immediately following extraction.

At days 1, 3, and 7, cardiac blood was centrifuged to collect serum and then analyzed for liver enzymes by the Penn Vet Clinical Pathology Diagnostic Lab. At day 1, urine ( $n=1$ mouse) was examined by TEM imaging; $5 \mu \mathrm{L}$ of urine was diluted 1:1 with water and placed onto a TEM grid for 3 minutes, then grids were lightly blotted, allowed to air-dry. Bright-field TEM imaging was performed on a Tecnai T12 microscope. Dark-field imaging and energy-dispersive X-ray spectroscopy (EDX) were performed by the Singh Center for Nanotechnology using a JEOL NEOARM.

Feces, urine, and organ samples were stored at $-20^{\circ} \mathrm{C}$, then weighed and then analyzed by ICP-OES as described, by digestion in $1 \mathrm{~mL}$ aqua regia for a minimum of 12 hours. The percentage of injected dose per gram tissue (\%ID/g) was calculated based on the mass of tissue that was harvested and processed for ICP analysis, and which often comprised a subset of the total organ. Where liver and spleen data is expressed as percent injected dose (\%ID) for the purpose of comparison with other studies, values were calculated based on approximate intact organ weight; this was estimated using published datasets of female 8 -week-old C57BL $/ 6$ mice, with values of liver mass $=5 \%$ of total body mass and spleen mass $=0.4 \%$ of total body mass (Mouse Phenotype Database, Jackson Laboratory).

Several tissue samples were reserved for histological evaluation at 1 day, 1 week, and 3 months: kidney $(n=3$ per timepoint), liver $(n=3)$, spleen $(n=3)$, and lung $(n=1)$. One experimentally naïve mouse was used as a control. Sections of these tissues were fixed in formalin; paraffin embedding was performed by the Penn Center for Musculoskeletal Disorders Histology Core, while sectioning and staining was performed by the Penn Vet Comparative Histology Core. All samples were then imaged using an EVOS light microscope and examined for signs of pathology (apoptosis, immune cell infiltration, damage to tissue architecture, etc.). ${ }^{8}$ A subset of slides $(n=1$ mouse per timepoint, including control mouse $)$ were also examined by the Penn Vet Comparative Histology Core, and pathological evaluation was performed by a board-certified 
veterinary pathologist who was blinded to the experimental groups. At day 1 and day 7, separate liver tissue sections were also preserved for examination of ultrastructure by electron microscopy. These were suspended in $100 \mu \mathrm{L}$ of fixative solution containing $2 \%$ glutaraldehyde and $2 \%$ paraformaldehyde $(\mathrm{w} / \mathrm{v})$, and prepared for EM imaging by the University of Pennsylvania Electron Microscopy Resource Lab. Bright-field imaging was performed on a JEOL 1010 microscope. Darkfield imaging and energy-dispersive X-ray spectroscopy (EDX) were performed by the Singh Center for Nanotechnology using a JEOL NEOARM.

Statistical analysis: Statistical analysis was performed using one-way ANOVA with post-hoc Tukey HSD test, using Astatsa online software (http://astatsa.com).

\section{EXPERIMENTAL REFERENCES}

(1) Jadzinsky, P. D.; Calero, G.; Ackerson, C. J.; Bushnell, D. A.; Kornberg, R. D. Structure of a Thiol Monolayer-Protected Gold Nanoparticle at 1.1 Å Resolution. Science 2007, 318 (5849), 430-433.

(2) Hermanson, G. Bioconjugate Techniques, 1st ed.; Elsevier, 1996.

(3) Bachelder, E. M.; Beaudette, T. T.; Broaders, K. E.; Dashe, J.; Fréchet, J. M. J. Acetal-Derivatized Dextran: An Acid-Responsive Biodegradable Material for Therapeutic Applications. J. Am. Chem. Soc. 2008, 130 (32), 10494-10495.

(4) Brust, M.; Walker, M.; Bethell, D.; J. Schiffrin, D.; Whyman, R. Synthesis of Thiol-Derivatised Gold Nanoparticles in a TwoPhase Liquid-Liquid System. Journal of the Chemical Society, Chemical Communications 1994, 0 (7), 801-802.

(5) Higbee-Dempsey, E.; Amirshaghaghi, A.; Case, M. J.; Miller, J.; Busch, T. M.; Tsourkas, A. Indocyanine Green-Coated Gold Nanoclusters for Photoacoustic Imaging and Photothermal Therapy. Advanced Therapeutics 2019, 0 (0), 1900088.

(6) Al Zaki, A.; Joh, D.; Cheng, Z.; De Barros, A. L. B.; Kao, G.; Dorsey, J.; Tsourkas, A. Gold-Loaded Polymeric Micelles for Computed Tomography-Guided Radiation Therapy Treatment and Radiosensitization. ACS Nano 2014, 8 (1), $104-112$.

(7) Zaki, A. A.; Hui, J. Z.; Higbee, E.; Tsourkas, A. Biodistribution, Clearance, and Toxicology of Polymeric Micelles Loaded with 0.9 or 5 Nm Gold Nanoparticles. J Biomed Nanotechnol 2015, 11 (10), 1836-1846.

(8) Ibrahim, K. E.; Al-Mutary, M. G.; Bakhiet, A. O.; Khan, H. A. Histopathology of the Liver, Kidney, and Spleen of Mice Exposed to Gold Nanoparticles. Molecules 2018, 23 (8). 


\section{SUPPORTING FIGURES}

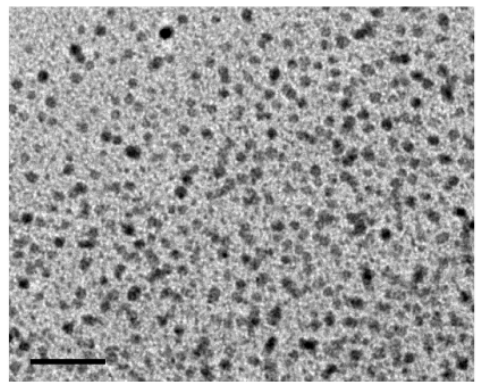

Figure S1. Electron microscopy image of Dextran-AuNPs, suspended in water and dried on a grid. Scale bar $=20 \mathrm{~nm}$.

A

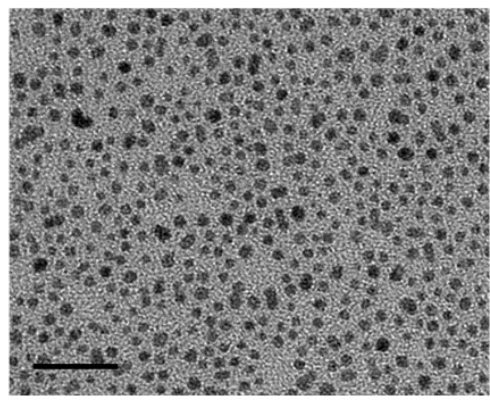

B

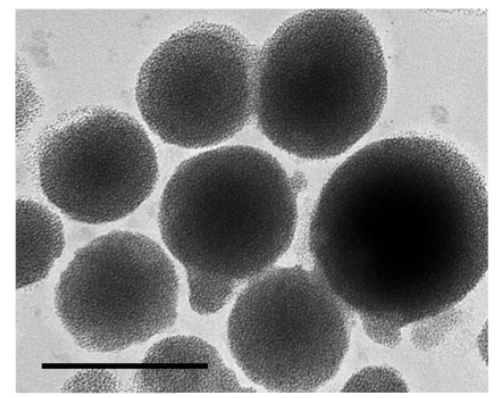

Figure S2. (a) Electron microscopy image of dodecanethiol-coated AuNPs (“C $\mathrm{C}_{12}$-AuNPs"), suspended in toluene and dried on a grid. Scale bar $=20 \mathrm{~nm}$. (b) Electron microscopy image of " $\mathrm{C}_{12}$-AuNPs-Micelles", formed by encapsulating $\mathrm{C}_{12}$ AuNP clusters with PEG-PCL. Micelles were suspended in water and dried on a grid. Scale bar $=200 \mathrm{~nm}$. 

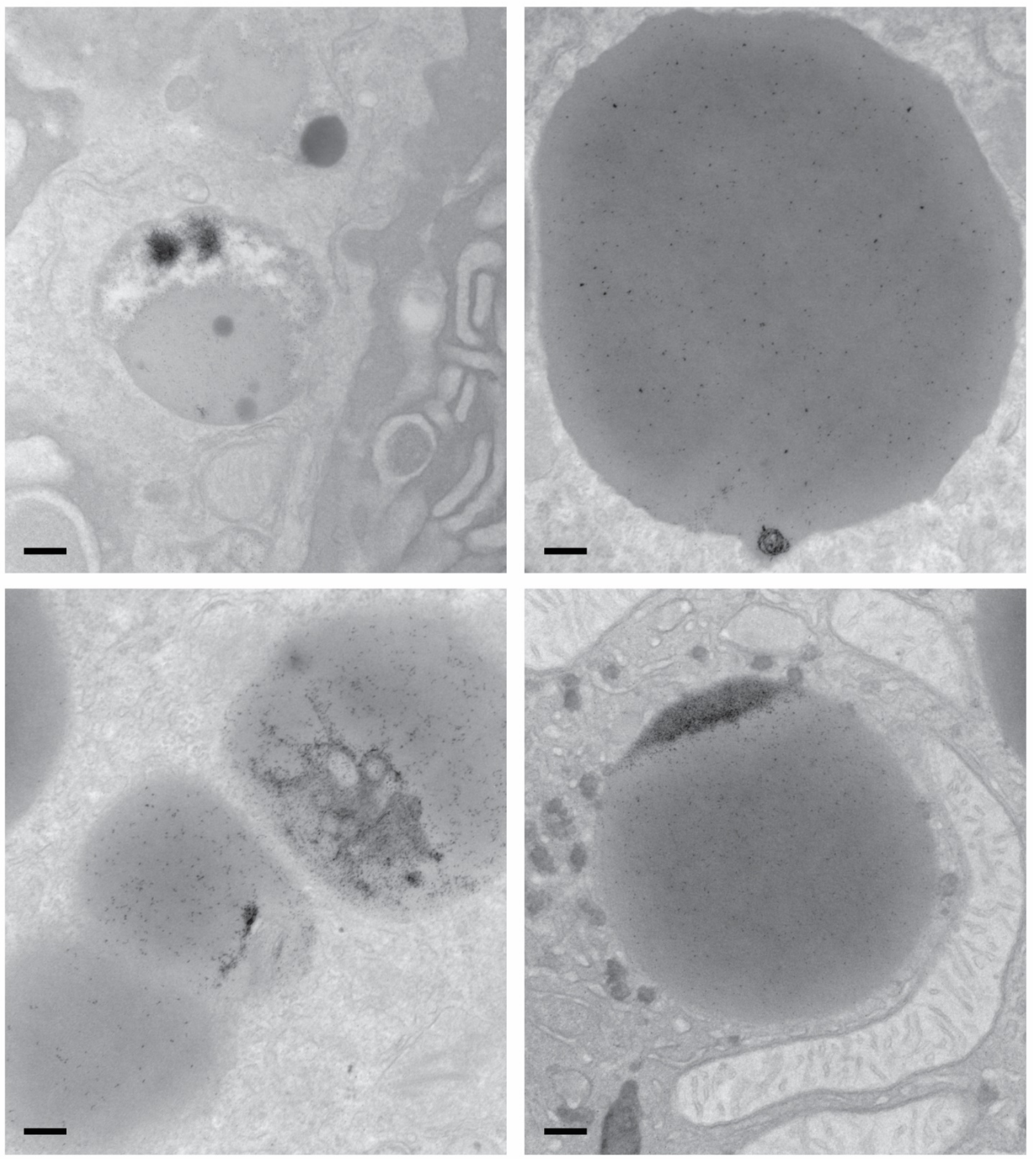

Figure S3. Electron microscopy of mouse liver at 24 hours post-injection. Mice were injected intravenously with AcetalDextran-AuNP-Micelles ("ADAMs") at 100 mg Au per kgbody weight. Liver samples were harvested at 24 hours postinjection and tissue was fixed and sectioned for electron microscopy imaging. Scale bar $=200 \mathrm{~nm}$. 
A

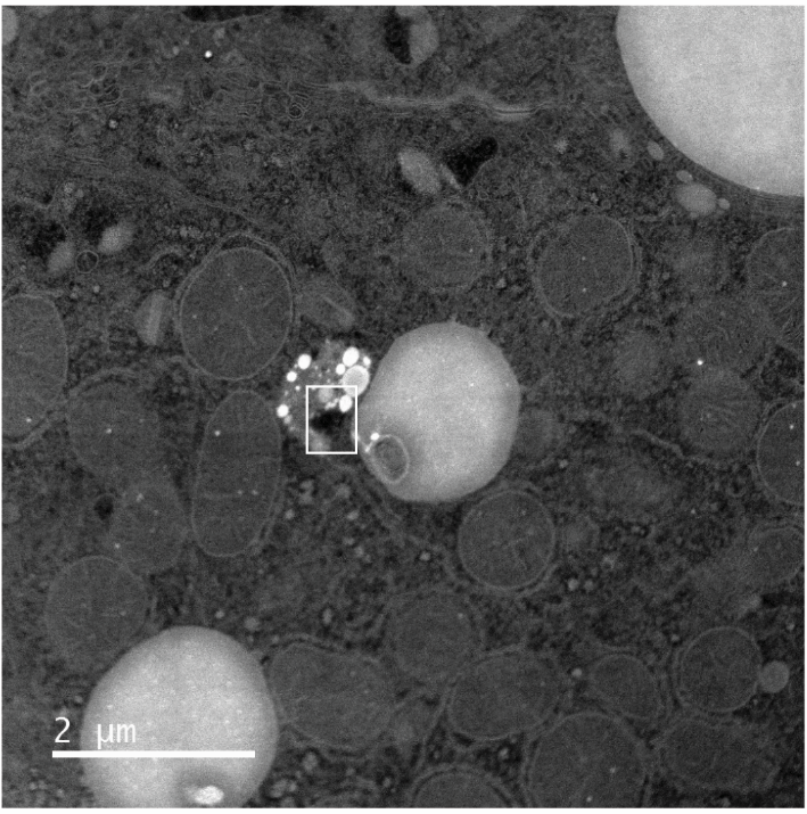

B

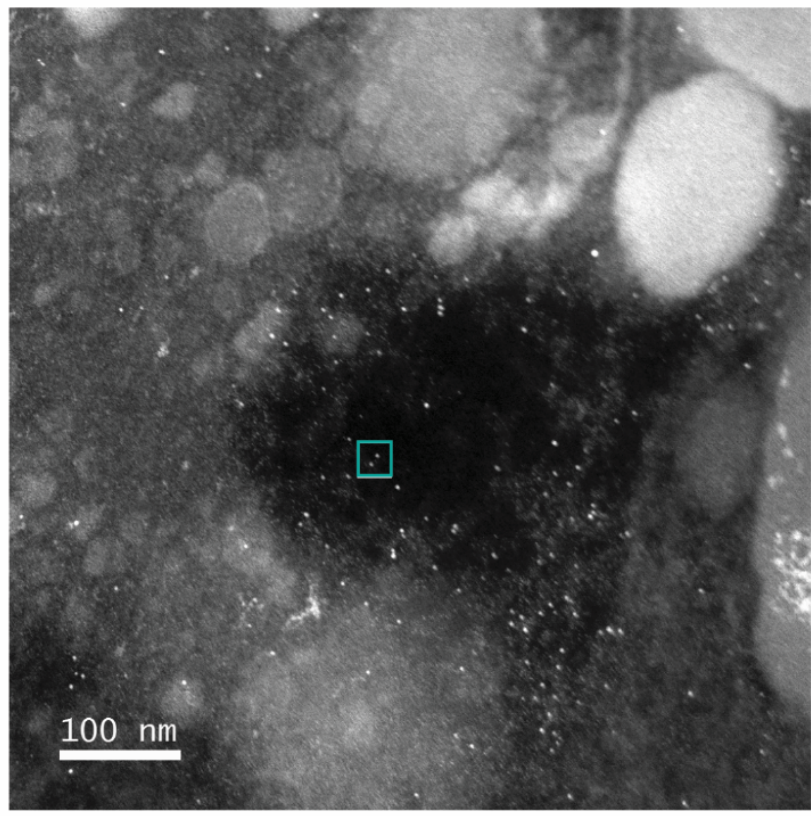

C

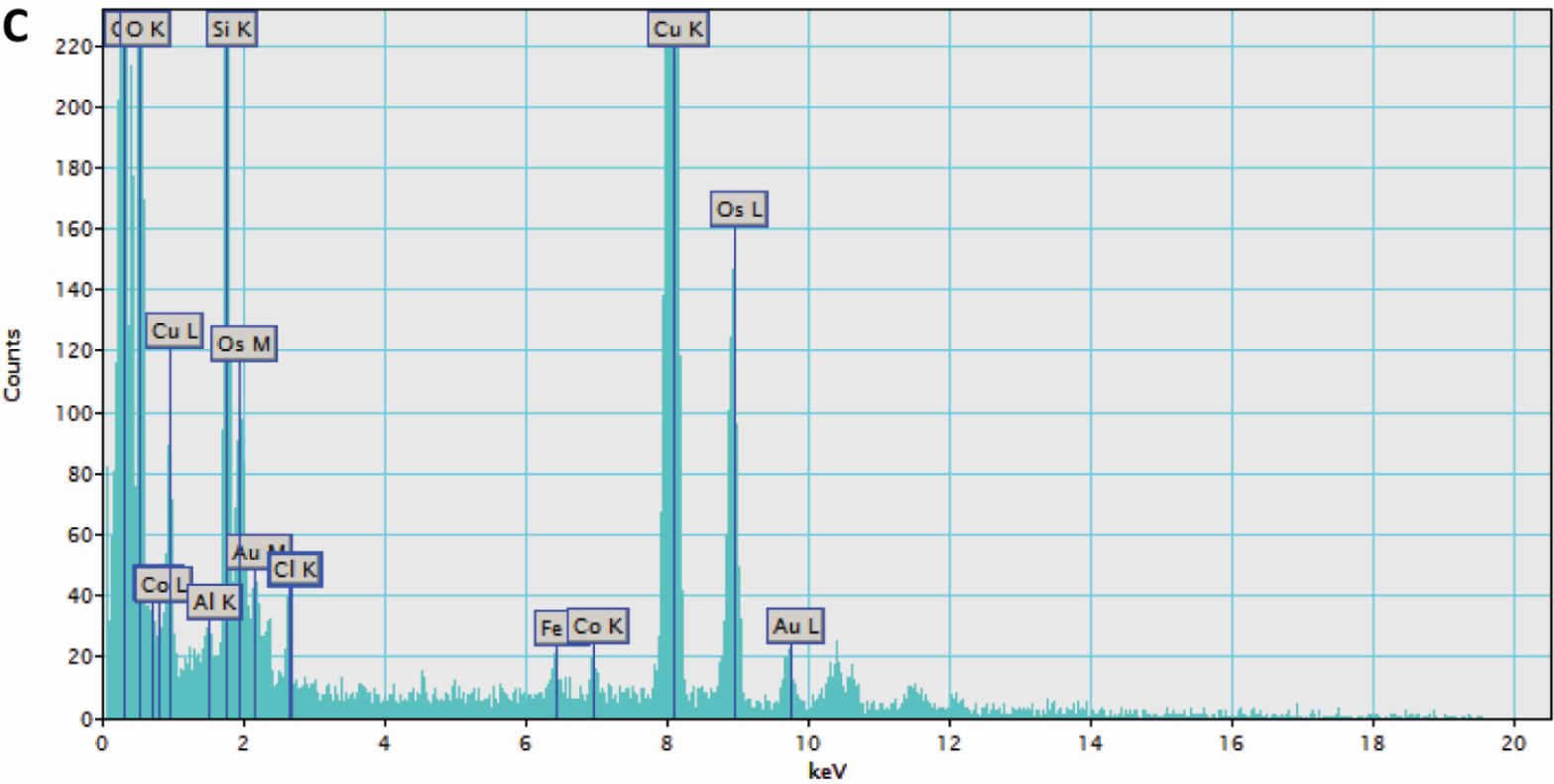

Figure S4. Electron microscopy and energy-dispersive X-ray spectroscopy (EDX) of mouse liver. Mice were injected intravenously with Acetal-Dextran-AuNP-Micelles ("ADAMs") at $100 \mathrm{mg}$ Au per kg body weight. Liver samples were harvested at 24 hours post-injection and tissue was fixed and sectioned for electron microscopy imaging. (a) Dark-field electron microscopy; white box indicates area magnified in (b). (b) Dark-field electron microscopy; turquoise box indicates area used for EDX. (c) EDX analysis confirmed the presence of elemental gold. 

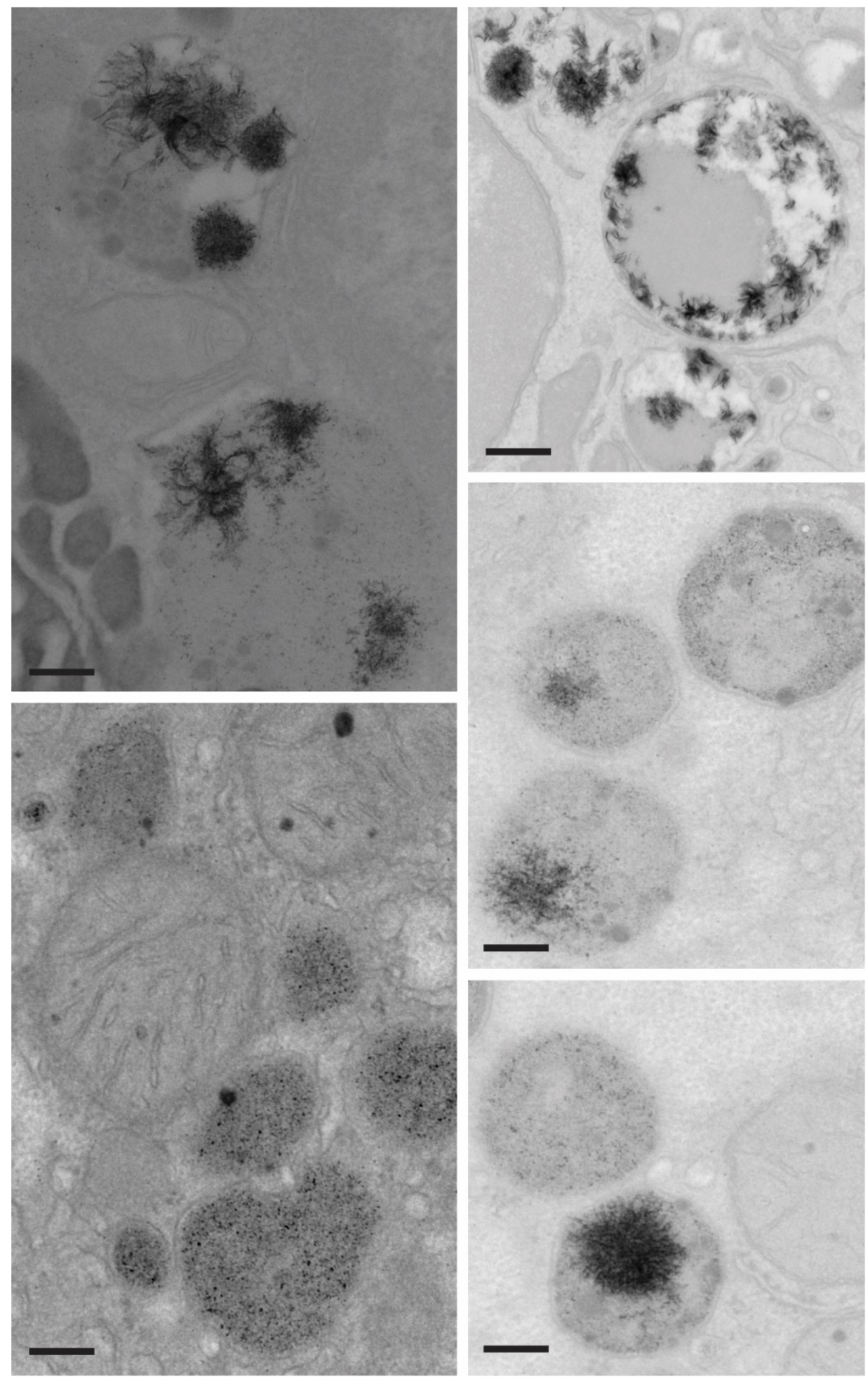

Figure S5. Electron microscopy of mouse liver at 7 days post-injection. Mice were injected intravenously with AcetalDextran-AuNP-Micelles ("ADAMs") at $100 \mathrm{mg}$ Au per kg body weight. Liver samples were harvested at 7 days post-injection and tissue was fixed and sectioned for electron microscopy imaging. Scale bar $=200 \mathrm{~nm}$. 


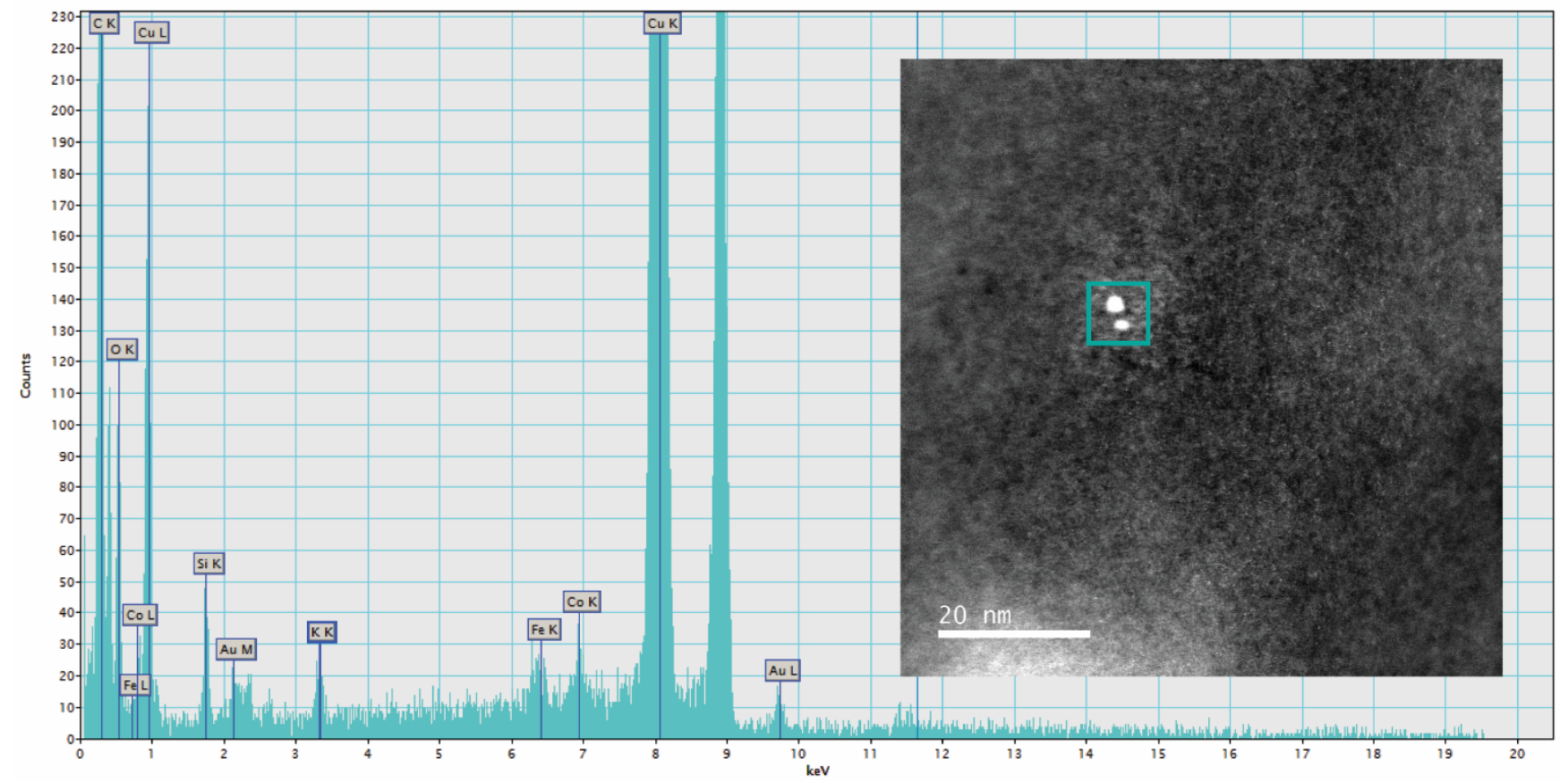

Figure S6. Energy-dispersive X-ray spectroscopy (EDX) and electron microscopy of mouse urine. Mice were injected intravenously with AcetalDextran-AuNP-Micelles ("ADAMs") at $100 \mathrm{mg} \mathrm{Au} \mathrm{per} \mathrm{kg} \mathrm{body} \mathrm{weight.} \mathrm{Urine} \mathrm{samples} \mathrm{were} \mathrm{col-}$ lected directly from mice at 24 hours post-injection, diluted 1:1 in pure water, and dried on a grid for electron microscopy imaging. EDX analysis confirmed the presence of elemental gold. Inset: Dark-field electron microscopy; teal box indicates area used for EDX.

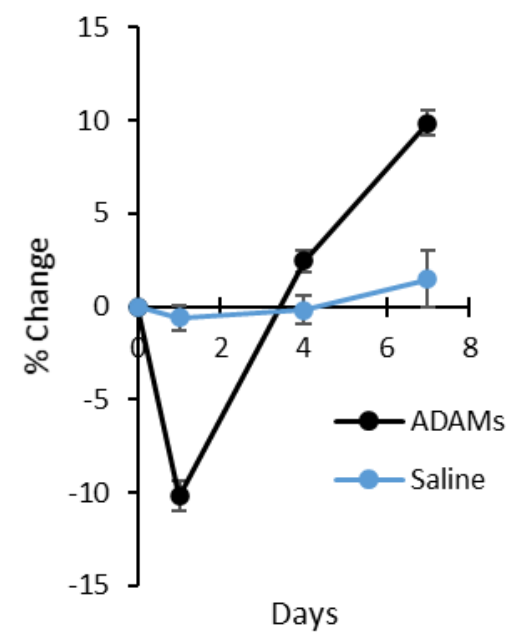

Figure S7. Changes in mouse body weight after intravenous injection with either saline or AcetalDextran-AuNP-Micelles ("ADAMs", $100 \mathrm{mg}$ Au per kg body weight). ADAMs data is replicated in Figure 7a. Data is expressed as percent change in mouse body weight over time, normalized to pre-injection weight. $n=3$ mice per group, \pm standard error of the mean. 
NMR SPECTRA

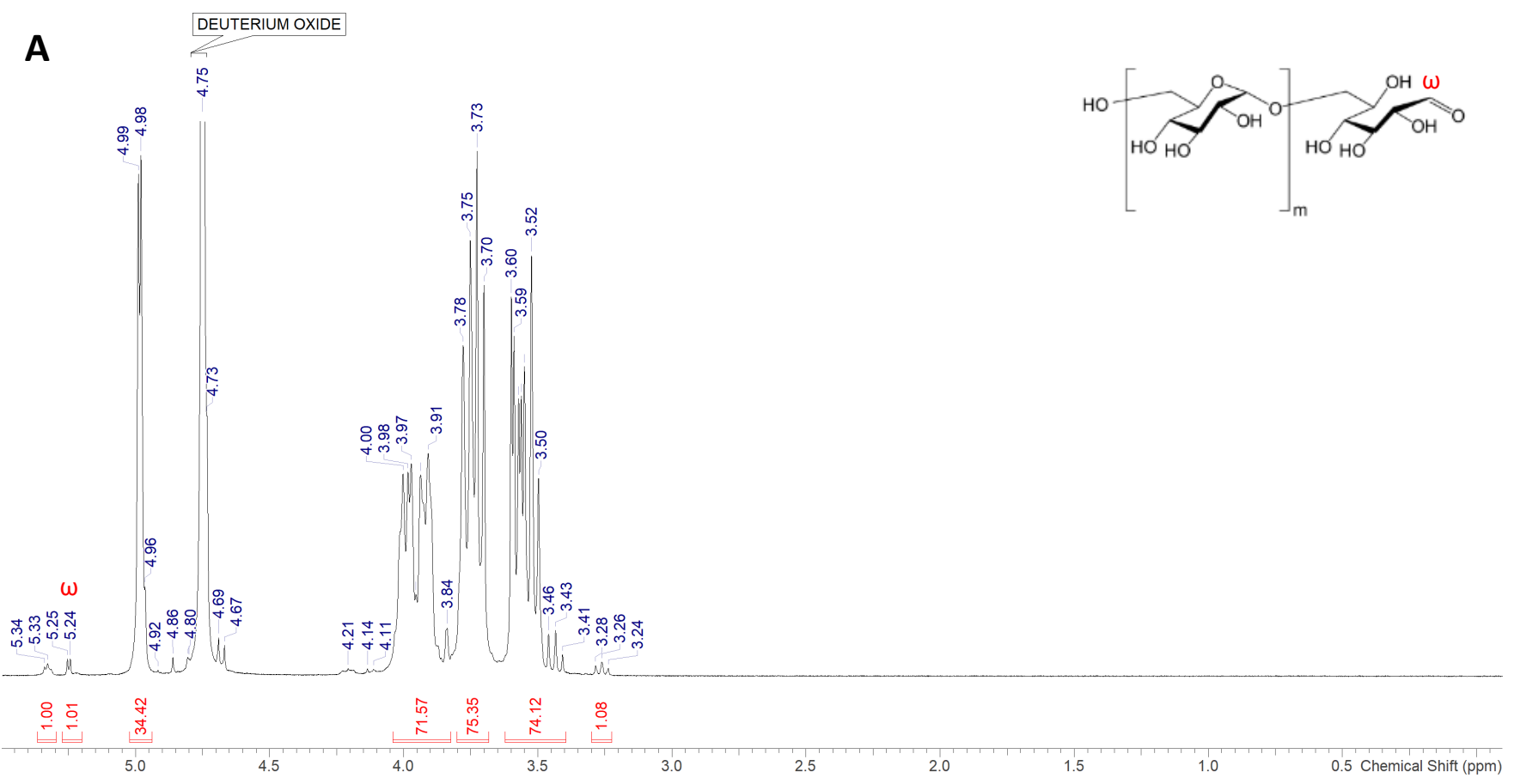

(a) ${ }^{1} \mathrm{H}$ NMR of dextran, M.W. $5000 \mathrm{Da},-0.1$ to $5.5 \mathrm{ppm}\left(\mathrm{D}_{2} \mathrm{O}, 360 \mathrm{MHz}\right)$. $\omega$ indicates terminal aldehyde group.

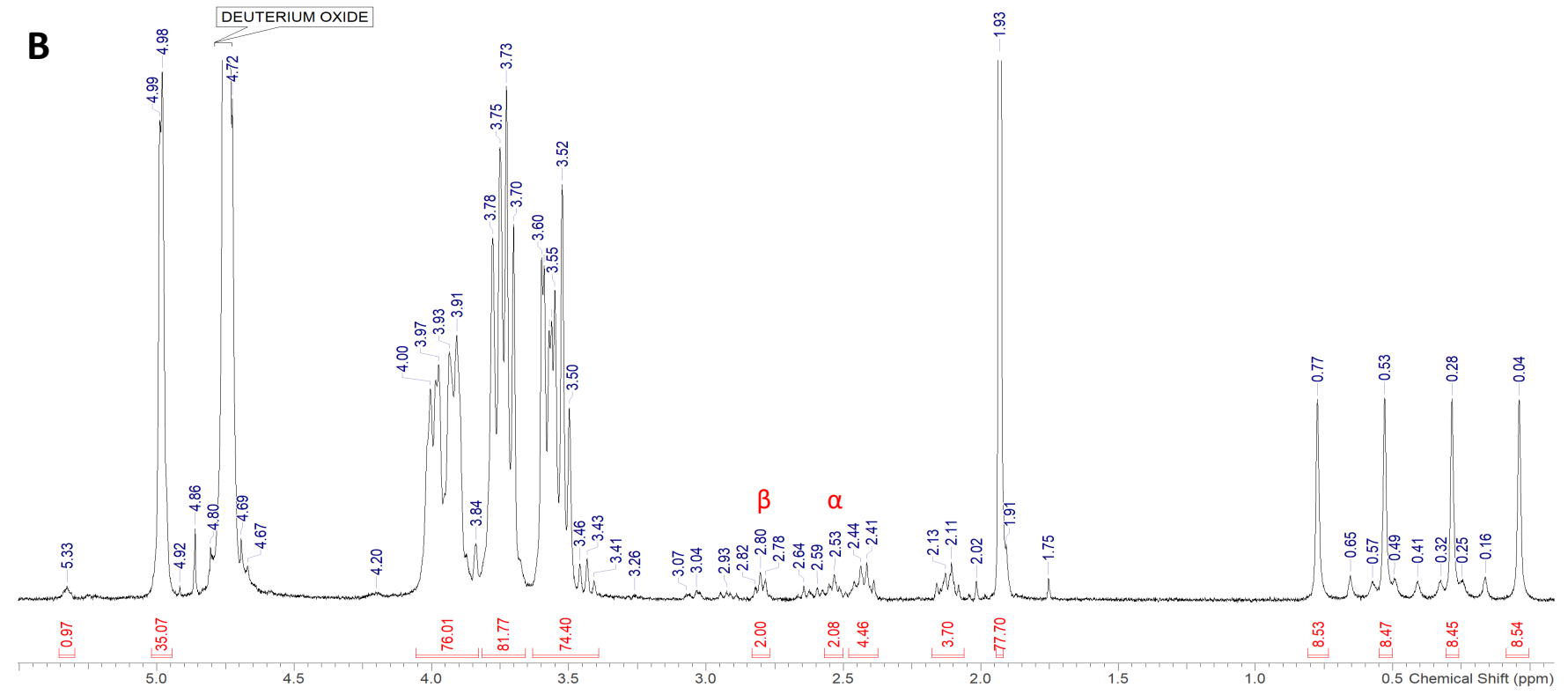



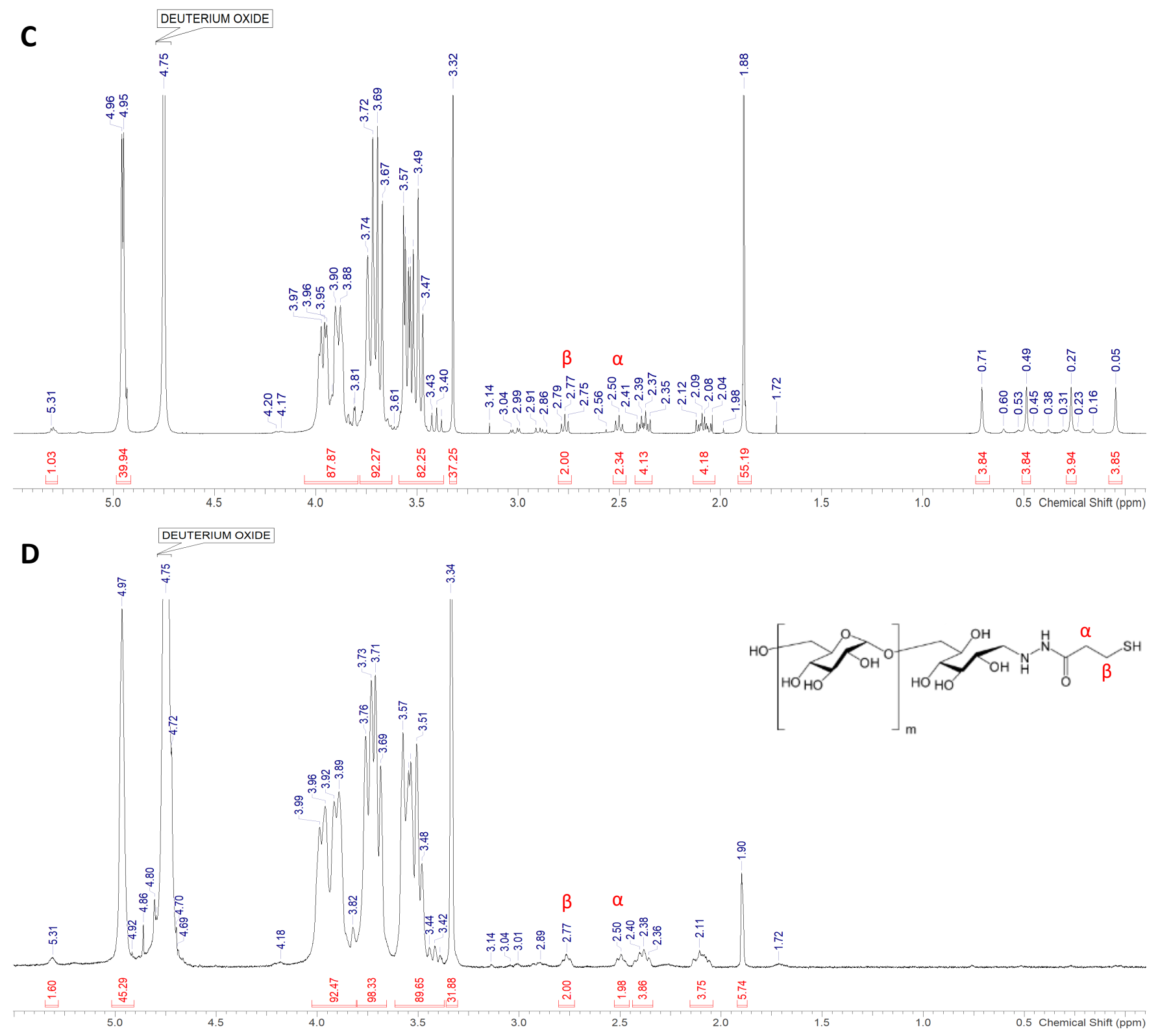

(b-d) ${ }^{1} \mathrm{H}$ NMR of thiolated dextran, -0.1 to $5.5 \mathrm{ppm}$. Successful conjugation with 3,3'-dithiobis(propanoic dihydrazide) was confirmed by disappearance of peaks at $\sim 5.25 \mathrm{ppm}$ (dextran's terminal aldehyde, $-\mathrm{CHOH}$-CHO) accompanied by emergence of new peaks at $\sim 2.51$ and $\sim 2.78 \mathrm{ppm}\left(-\mathrm{NH}-\mathrm{CO}-\mathrm{CH}_{2}-\mathrm{CH}_{2}-\mathrm{SH}\right.$, respectively). ${ }^{1,2}$ (b) Reaction milieu, before purification $\left(\mathrm{D}_{2} \mathrm{O}, 360 \mathrm{MHz}\right)$. (c) After purification via four washes in $90 \%$ methanol $\left(\mathrm{D}_{2} \mathrm{O}, 300 \mathrm{MHz}\right)$. (d) After alternative purification via Soxhlet extraction in $100 \%$ methanol $\left(\mathrm{D}_{2} \mathrm{O}, 360 \mathrm{MHz}\right)$. Reaction contaminants include cyanoborohydride (0.04-0.77 ppm), ${ }^{3}$ TCEP/TCEPO $(2.04-2.12 ; 2.35-2.41 \mathrm{ppm}),{ }^{4,5}$ and methanol (3.33 ppm). 


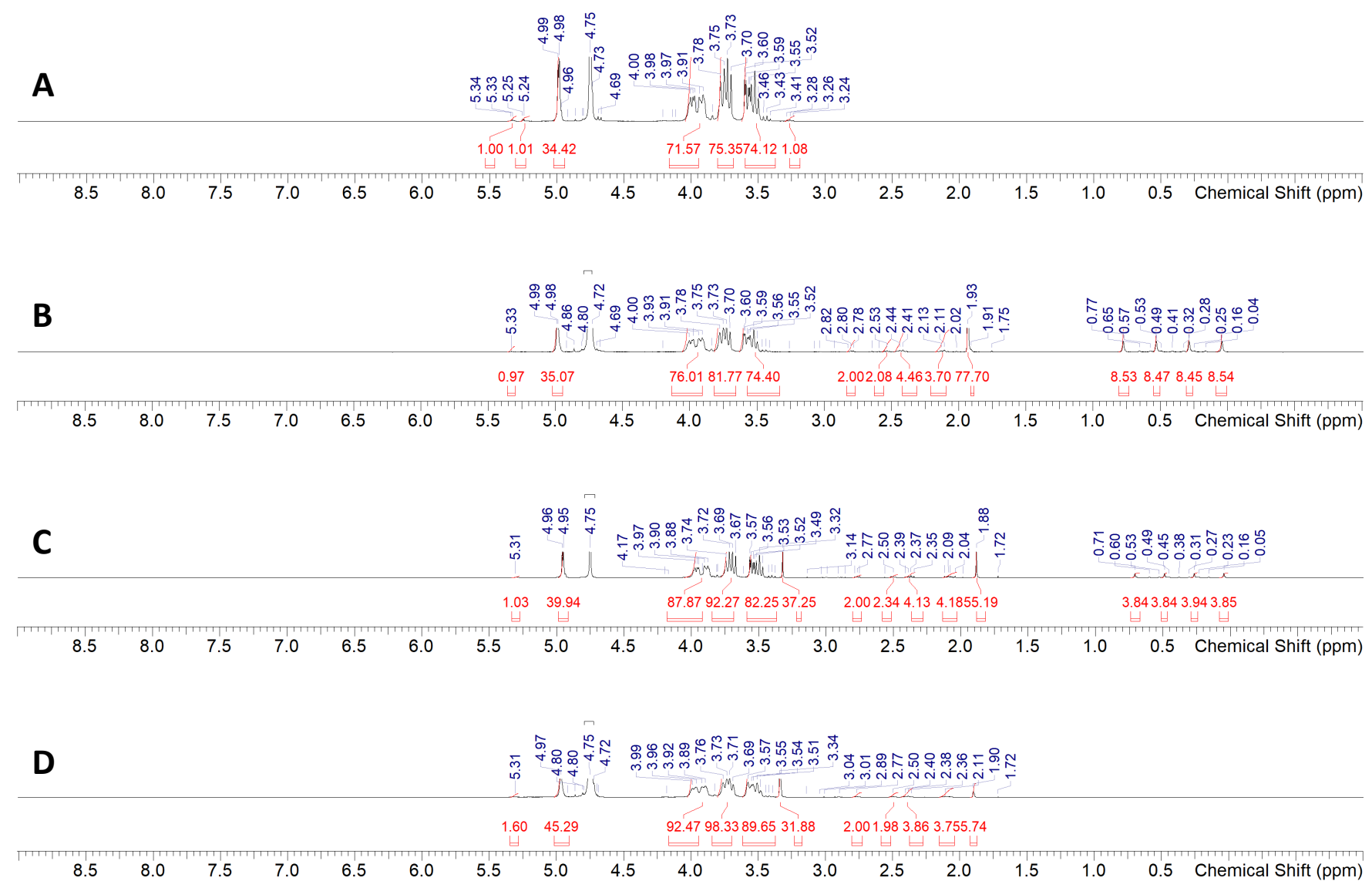

(a-d) All spectra, -1 to $9 \mathrm{ppm}$.

\section{FIGURE REFERENCES}

(1) Shu, X. Z.; Liu, Y.; Luo, Y.; Roberts, M. C.; Prestwich, G. D. Disulfide Cross-Linked Hyaluronan Hydrogels. Biomacromolecules 2002, 3 (6), 1304-1311.

(2) Ossipov, D. A.; Yang, X.; Varghese, O.; Kootala, S.; Hilborn, J. Modular Approach to Functional Hyaluronic Acid Hydrogels Using Orthogonal Chemical Reactions. Chem. Commun. 2010, 46 (44), 8368-8370.

(3) Ruman, T.; Kuśnierz, A.; Jurkiewicz, A.; Leś, A.; Rode, W. The Synthesis, Reactivity and 1H NMR Investigation of the Hydroxyborohydride Anion. Inorganic Chemistry Communications 2007, 10 (9), 1074-1078.

(4) Krezel, A.; Latajka, R.; Bujacz, G. D.; Bal, W. Coordination Properties of Tris(2-Carboxyethyl)Phosphine, a Newly Introduced Thiol Reductant, and Its Oxide. Inorg Chem 2003, 42 (6), 1994-2003.

(5) Tarnowski, M.; Barozet, A.; Johansson, C.; Eriksson, P.-O.; Engkvist, O.; Walsh, J.; Nissink, J. W. M. Utility of Resazurin, Horseradish Peroxidase, and NMR Assays to Identify Redox-Related False-Positive Behavior in High-Throughput Screens. Assay Drug Dev Technol 2018, 16 (3), 171-191. 


\section{DETAILED HISTOPATHOLOGY ANALYSIS}

(experimental groups have been un-blinded here)

1. Mouse: Control

- Liver:

- Mixed inflammatory cell infiltrates, hepatic sinusoids and portal regions, multifocal random, mild with occasional acute hepatocellular necrosis/apoptosis.

- Hepatocellular glycogen accumulation, diffuse, mild to moderate.

○ No nanoparticle-containing cells detected.

- Spleen:

- Extramedullary hematopoiesis, red pulp, diffuse, mild.

- No nanoparticle-containing cells detected.

- Kidney:

○ No significant findings.

- No nanoparticle-containing cells detected.

- Lung:

- Mononuclear peribronchiolar inflammatory cell infiltrates, multifocal, minimal.

○ Parenchymal collapse and atelectasis, multifocal, mild.

- No nanoparticle-containing cells detected.

2. Mouse: 1-day post-injection

- Liver:

- Mixed inflammatory cell infiltrates, hepatic sinusoids and portal regions, multifocal random, minimal to mild with rare acute hepatocellular necrosis/apoptosis.

$\circ \quad$ 110 nanoparticle-containing cells in 5 high powered fields (40x).

- Cells with nanoparticles are overall smaller than in Mouse:3-months, with less cytoplasmic nanoparticles, and nanoparticles have slightly different tinctorial properties, being slightly more yellow rather than pale green.

- Spleen:

○ Up to 274 nanoparticle-containing cells in a single high powered field (40x) (mostly in the red pulp).

- Cells with nanoparticles are overall smaller than in Mouse:3-months, with less cytoplasmic nanoparticles, and nanoparticles have slightly different tinctorial properties, being slightly less bright yellow/golden.

$\bigcirc \quad$ Extramedullary hematopoiesis, red pulp, diffuse, mild.

- Kidney:

$\circ$ No significant findings and no nanoparticle-containing cells seen.

- Lung:

- Parenchymal collapse and atelectasis, multifocal, mild to moderate.

- No nanoparticle-containing cells detected.

3. Mouse: 1-week post-injection

- Liver:

- Mixed inflammatory cell infiltrates, hepatic sinusoids and portal regions, multifocal random, minimal to mild with occasional acute hepatocellular necrosis/apoptosis and few nanoparticle-containing cells. 
○ 83 nanoparticle-containing cells in 5 high powered fields (40x). (In the hepatic sinusoids and lesser portal regions).

- Cells with nanoparticles are overall smaller than in Mouse:3-months, with less cytoplasmic nanoparticles, however, nanoparticles share similar features as mice B17-16-1 and BM3, being variably golden/yellow or pale green.

○ Hepatocellular glycogen accumulation, diffuse, mild.

- Spleen:

○ 180 nanoparticle-containing cells in a single high powered field (40x).

- Cells with nanoparticle exhibit tinctorial properties similar to both mice Mouse:3-months and Mouse:1-day

- Extramedullary hematopoiesis, red pulp, diffuse, mild.

- Kidney:

- 21 nanoparticle-containing cells in 10 high powered fields (40x). (Exclusively in the interstitium)

$\circ$ No significant findings.

- Lung:

- Parenchymal collapse and atelectasis, multifocal, minimal.

○ No nanoparticle-containing cells detected.

4. Mouse: 3-months post-injection

- Liver:

- Mixed inflammatory cell infiltrates, hepatic sinusoids and portal regions, multifocal random, mild with occasional acute hepatocellular necrosis/apoptosis and few nanoparticle-containing cells.

$\circ \quad$ 35 nanoparticle-containing cells in 5 high powered fields (40x). (In the hepatic sinusoids and portal regions).

○ Hepatocellular glycogen accumulation, diffuse, mild.

- Spleen: (Limited splenic tissue available for evaluation)

$\circ$ Up to $\sim 150$ nanoparticle-containing cells in a single high powered field (40x) (mostly in the red pulp).

- Extramedullary hematopoiesis, red pulp, diffuse, mild.

- Kidney:

$\circ \sim 10$ nanoparticle-containing cells in 10 high powered fields (40x). (Exclusively in the interstitium)

○ No significant findings.

- Lung:

- Mononuclear peribronchiolar inflammatory cell infiltrates, multifocal, minimal.

- Parenchymal collapse and atelectasis, multifocal, mild to moderate.

○ No nanoparticle-containing cells detected.

Comments:

- Cells containing small globular particles (the presumed gold nanoparticles) are seen in 3 of the 4 mice. MouseControl didn't have any particles in any of the organs and is therefore considered as the control mouse. In all organs, the nanoparticles are suspected to accumulate in macrophages/monocytes, such as Kupffer cells or circulating macrophages/monocytes in the hepatic sinusoids, red pulp macrophages in the spleen and interstitial macrophages in the kidney.

- The nanoparticles in the liver of Mouse:3-months were gray/green and more obviously expanded the cells (up to $20 \mu \mathrm{m}$ diameter) than in Mouse:1-day and Mouse:1-week. In Mouse:1-day, the particles were more yellow/golden whereas Mouse: 1-week had a combination of both gray/green and yellow/golden particles. In general, the nanoparticle-containing cells were not associated with any inflammation or parenchymal damage. The few cells seen with inflammatory cell infiltrates in Mouse:3-months and Mouse:1-week are most likely not causative for the inflammation. 
- Similarly, in the spleen, nanoparticles exhibit different tinctorial properties from one mouse to the other. The kidney has the lowest density of nanoparticles, which are only seen in cells within the interstitium. No nanoparticles were seen in any of the samples of lung.

- Additional findings, such as the multifocal inflammatory cell infiltrates in the liver and peribronchial tissue of the lung, as well as the extramedullary hematopoiesis in the spleen, are commonly seen in control mice and are considered as a normal background finding.

References:

- Comparative Anatomy and Histology - A mouse and Human Atlas. 2012. Treuting PM, Dintzis SM, editors. London, Academic Press.

- INHAND: Proliferative and nonproliferative lesions of the rat and mouse hepatobiliary system. Thoolen B, Maronpot RR, Harada T, Nyska A, Rousseaux C, Nolte T, Malarkey DE, Kaufmann W, Küttler K, Deschl U, Nakae D, Gregson R, Vinlove MP, Brix AE, Singh B, Belpoggi F, Ward JM. Toxicol Pathol. 2010 Dec;38(7 Suppl):5S-81S.

- INHAND: Proliferative and nonproliferative lesions of the rat and mouse respiratory tract. Renne R, Brix A, Harkema J, Herbert R, Kittel B, Lewis D, March T, Nagano K, Pino M, Rittinghausen S, Rosenbruch M, Tellier P, Wohrmann T. Toxicol Pathol. 2009 Dec;37(7 Suppl):5S-73S.

- Pathology of laboratory rodents and rabbits, 4th ed. 2016. Percy DH, Barthold SW, editors. Ames (IA), Wiley-Blackwell. 